\title{
A Remark on Spectral Properties of Certain Non-selfadjoint Schrödinger Operators
}

\author{
Daisuke AIBA
}

Gakushuin University

(Communicated by F. Nakano)

\begin{abstract}
In this paper, we study the spectral and pseudospectral properties of the differential operator $H_{\varepsilon}=$ $-\partial_{x}^{2}+x^{2 m}+i \varepsilon^{-1} f(x)$ on $L^{2}(\mathbf{R})$, where $\varepsilon>0$ is a small parameter, $m \in \mathbf{N}$ and $f$ is a real-valued Morse function which satisfies $\left|\partial_{x}^{l}\left(f(x)-|x|^{-k}\right)\right| \leq C|x|^{-k-l-1}$ for $l=0,1,2,3$ and large $|x|$. We show that $\Psi(\varepsilon)=$ $\left(\sup _{\lambda \in \mathbf{R}}\left\|\left(H_{\varepsilon}-i \lambda\right)^{-1}\right\|\right)^{-1}$ and $\Sigma(\varepsilon)=\inf \Re\left(\sigma\left(H_{\varepsilon}\right)\right)$ satisfy $C^{-1} \varepsilon^{-v(m)} \leq \Psi(\varepsilon) \leq C \varepsilon^{-v(m)}$ and $\Sigma(\varepsilon) \geq$ $C^{-1} \varepsilon^{-v(m)}, v(m)=\min \left\{\frac{2 m}{k+3 m+1}, \frac{1}{2}\right\}$. This extends the result of I. Gallagher, T. Gallay and F. Nier [3] (2009) for the case $m=1$ to general $m \in \mathbf{N}$.
\end{abstract}

\section{Introduction}

We consider Schrödinger operator with a complex potential $\tilde{H}_{\varepsilon}=-\partial_{x}^{2}+x^{2}+i \varepsilon^{-1} f(x)$ in $L^{2}(\mathbf{R})$ where $\varepsilon>0$ is a small parameter and $f(x)$ is a real-valued function. In [ICM 2], C. Villani asked the following question: What is the condition on $f(x)$ for $\tilde{\Sigma}(\varepsilon)=$ $\inf \Re\left(\sigma\left(\tilde{H}_{\varepsilon}\right)\right) \rightarrow+\infty$ as $\varepsilon \rightarrow 0$ and how the rate of divergence ? In [5], J. H. Schenker has proved that $\tilde{\Sigma}(\varepsilon) \rightarrow+\infty$ as $\varepsilon \rightarrow 0$ if $L_{t} \stackrel{\text { def }}{=}\{x \in \mathbf{R} ; f(x)=t\}$ is essentially nowhere dense for each $t \in \mathbf{R}$. Now, we say that a set $S$ is essentially nowhere dense if $S=S^{\prime} \cup N$ where $S^{\prime}$ is nowhere dense and $N$ has Lebesgue measure zero. In [3], I. Gallagher, T. Gallay and F. Nier have studied the rate of growth of $\tilde{\Sigma}(\varepsilon)$ and the spectral quantity $\tilde{\Psi}(\varepsilon)=\left(\sup _{\lambda \in \mathbf{R}} \|\left(\tilde{H}_{\varepsilon}-\right.\right.$ $\left.i \lambda)^{-1} \|\right)^{-1}$ under the condition that $f(x)$ is a real-valued Morse function.

In this paper, we study the same problem for $H_{\varepsilon}=-\partial_{x}^{2}+x^{2 m}+i \varepsilon^{-1} f(x)$ where $m \geq 1$ is an integer. We consider the operator $H_{\varepsilon}$ with domain $\mathcal{D}=\left\{u \in H^{2}(\mathbf{R}) ; x^{2 m} u \in L^{2}(\mathbf{R})\right\}$. Let $H_{\infty}=-\partial_{x}^{2}+x^{2 m}$ be a self-adjoint operator with domain $\mathcal{D}$. Then $H_{\varepsilon}$ is $H_{\infty}$-bounded and skew-symmetric. Since $H_{\varepsilon}$ has a compact resolvent, the spectrum $\sigma\left(H_{\varepsilon}\right)$ consists of a countable number discrete eigenvalues $\left\{\lambda_{n}(\varepsilon)\right\}_{n \in \mathbf{N}}$ with $\Re\left(\lambda_{n}(\varepsilon)\right) \rightarrow+\infty$ as $n \rightarrow \infty$. The numerical range $\Theta\left(H_{\varepsilon}\right)=\left\{\left\langle H_{\varepsilon} u, u\right\rangle_{L^{2}} ; u \in \mathcal{D},\|u\|_{L^{2}}=1\right\}$ is obviously contained in the rectangle $\mathcal{R}_{\varepsilon}=\left\{\lambda \in \mathbf{C} ; \mathfrak{R}(\lambda) \geq a_{0}, \varepsilon \Im(\lambda) \in \overline{f(\mathbf{R})}\right\}$ where $a_{0}=\inf \sigma\left(H_{\infty}\right)$. Hence, we have

Received April 23, 2012; revised June 18, 2012

Mathematics Subject Classification: 35P15 
$\lambda_{n}(\varepsilon) \in \Theta\left(H_{\varepsilon}\right) \subset \mathcal{R}_{\varepsilon}$ for all $n \in \mathbf{N}$ and all $\varepsilon>0$. It follows that the imaginary axis $i \mathbf{R}$ is contained in the resolvent set of $H_{\varepsilon}$. We define

$$
\Sigma(\varepsilon)=\inf \Re\left(\sigma\left(H_{\varepsilon}\right)\right)=\min _{n \in \mathbf{N}} \Re\left(\lambda_{n}(\varepsilon)\right), \quad \text { and } \quad \Psi(\varepsilon)=\left(\sup _{\lambda \in \mathbf{R}}\left\|\left(H_{\varepsilon}-i \lambda\right)^{-1}\right\|\right)^{-1} .
$$

As is proved by [3], we have $\Sigma(\varepsilon) \geq \Psi(\varepsilon) \geq a_{0}$. To state our main theorem, we set the following hypothesis.

Hypothesis 1. Assume that $f \in C^{3}(\mathbf{R}, \mathbf{R})$ has the following properties:

(i) All critical points of $f$ are nondegenerate, i.e., $f^{\prime}(x)=0$ implies $f^{\prime \prime}(x) \neq 0$,

(ii) There exist positive constants $C$ and $k$ such that, for all $x \in \mathbf{R}$ with $|x| \geq 1$,

$$
\left|\partial_{x}^{l}\left(f(x)-\frac{1}{|x|^{k}}\right)\right| \leq \frac{C}{|x|^{k+l+1}}, \quad \text { for } l=0,1,2,3 .
$$

The main theorem of this paper is the following.

THEOREM 1. Suppose that $f$ satisfies Hypothesis 1 . Then there exists $C>0$ such that, for all $0<\varepsilon \ll 1$,

$\frac{1}{C \varepsilon^{\nu(m)}} \leq \Psi(\varepsilon) \leq \frac{C}{\varepsilon^{\nu(m)}} \quad$ and $\quad \Sigma(\varepsilon) \geq \frac{1}{C \varepsilon^{\nu(m)}} \quad$ where $\quad v(m)=\min \left\{\frac{2 m}{k+3 m+1}, \frac{1}{2}\right\}$.

A few remarks are in order.

REMARK 1. For the case $m=1$, Theorem 1 was proven by I. Gallagher, T. Gallay and F. Nier [3]. Our result shows that $v(m)>v(n)$ if $m>n$.

REMARK 2. Since $\Theta\left(H_{\varepsilon}\right) \subset \mathcal{R}_{\varepsilon}, H_{\varepsilon}-a_{0}$ is maximal accretive and $H_{\varepsilon}$ is the infinitesimal generator of $C_{0}$-semigroup $e^{-t H_{\varepsilon}}$. We set that $C(\mu)=\frac{1}{\pi}\left\{\frac{\mu}{\tan \alpha} N(\mu)+\frac{2 \pi}{\sin \alpha}\right\}$ and $N(\mu)=\sup _{\lambda \in \mathbf{R}}\left\|\left(H_{\varepsilon}-\mu-i \lambda\right)^{-1}\right\|$ where the angle $\alpha$ satisfies $\tan (2 \alpha)=a_{0} \varepsilon\|f\|_{\infty}^{-1}$. As is proved by [3], for any $0<\mu<\Sigma(\varepsilon)$, we have $\left\|e^{-t H_{\varepsilon}}\right\| \leq C(\mu) e^{-\mu t}$ for all $t \geq 0$.

In spite of $\Sigma(\varepsilon) \geq \Psi(\varepsilon), \Sigma(\varepsilon)$ can be much bigger than $\Psi(\varepsilon)$ in some particular cases. The following is also a generalization of the Theorem 1.9 of [3].

THEOREM 2. Fix $k>0$ and set $f(x)=\left(1+x^{2}\right)^{-k / 2}$. Then there exists a constant $C>0$ such that for all $0<\varepsilon \ll 1$,

$$
\Sigma(\varepsilon) \geq \frac{C}{\varepsilon^{v^{\prime}(m)}}, \quad \text { where } \quad v^{\prime}(m)=\min \left\{\frac{1}{2}, \frac{2 m}{k+2 m}\right\} .
$$

The rest of the paper is devoted to the proof of Theorem 1 and Theorem 2 . Theorem 1 is proved in section 2 and Theorem 2 in section 3. Before going into the next, we remark that

(i) $\Psi(\varepsilon)>a_{0}$ if $f \in L^{\infty}(\mathbf{R})$ is not a constant, 
(ii) $\Psi(\varepsilon) \rightarrow \infty$ as $\varepsilon \rightarrow 0$ if $f \in L^{\infty}(\mathbf{R}) \cap C^{0}(\mathbf{R})$ and for any $t \in \mathbf{R}, L_{t}$ has empty interiors.

This can be proven similarly to Proposition 1.4 and Lemma 2.1 of [3]. Throughout this paper, we denote by $C$ various constants whose exact values are not important. Thus they may differ from one place to the other.

\section{Resolvent Estimates}

In this section, we prove Theorem 1 by using the localization techniques and semiclassical subelliptic estimates. The proof patterns after that of Proposition 4.1 of [3], and we shall point out only what modifications are necessary for the generalization. We estimate

$$
\kappa(\varepsilon, \lambda)=\left\|\left(H_{\varepsilon}-i \lambda\right)^{-1}\right\| \text { for } \lambda \in \mathbf{R} \text { and } 0<\varepsilon \ll 1 .
$$

Under Hypothesis $1, f$ has only a finite number of critical points, and we denote the set of critical values of $f$ by

$$
\operatorname{cv}(f)=\left\{f(x) ; x \in \mathbf{R}, f^{\prime}(x)=0\right\} .
$$

Proposition 1. Iff satisfies Hypothesis 1 . Then for any $\lambda \in \mathbf{R}$ and $0<\varepsilon \ll 1$, the quantity $\kappa(\varepsilon, \lambda)$ satisfies the following estimates:

(i) If $\operatorname{dist}(\varepsilon \lambda, f(\mathbf{R})) \geq \delta>0$, then $\kappa(\varepsilon, \lambda) \leq \varepsilon / \delta$.

(ii) If $\operatorname{dist}(\varepsilon \lambda, \operatorname{cv}(f) \cup\{0\}) \geq \delta>0$, then $\kappa(\varepsilon, \lambda) \leq C_{\delta} \varepsilon^{2 / 3}$.

(iii) If $\lambda=\lambda(\varepsilon)$ is such that $\lim _{\varepsilon \rightarrow 0} \varepsilon \lambda(\varepsilon)=\alpha \in \operatorname{cv}(f) \backslash\{0\}$, then

$$
\varlimsup_{\varepsilon \rightarrow 0} \varepsilon^{-1 / 2} \kappa(\varepsilon, \lambda(\varepsilon)) \leq C .
$$

(iv) For $\lambda=0$, the quantity $\kappa(\varepsilon, 0)$ satisfies

$$
\kappa(\varepsilon, 0) \leq \begin{cases}C \varepsilon^{\frac{2 m}{k+2 m}}, & \text { if } 0 \notin f(\mathbf{R}), \\ C \varepsilon^{\min \left\{\frac{2 m}{k+2 m}, \frac{2}{3}\right\}}, & \text { if } 0 \in f(\mathbf{R}) \backslash \operatorname{cv}(f), \\ C \varepsilon^{\min \left\{\frac{2 m}{k+2 m}, \frac{1}{2}\right\}}, & \text { if } 0 \in \operatorname{cv}(f) .\end{cases}
$$

(v) There exists $C>1$ such that, for all $\lambda \in \mathbf{R}$ and $0<\varepsilon \ll 1$,

$$
\kappa(\varepsilon, \lambda) \leq C \varepsilon^{\nu(m)} . \quad \text { where } v(m)=\min \left\{\frac{2 m}{k+3 m+1}, \frac{1}{2}\right\} .
$$

For the proof of Proposition 1, we use the following localization scheme. The proof of the following two lemmas may be found in [3].

LEMMA 1. Let $Q=-\Delta+V$ in $\mathbf{R}^{d}$, where $V$ is a complex valued measurable function. Let $\left\{\chi_{j}^{2}\right\}_{j \in J}$, where $\chi_{j} \in C_{0}^{\infty}\left(\mathbf{R}^{d}, \mathbf{R}\right)$ be such that

$$
\sum_{j \in J} \chi_{j}(x)^{2}=1, \quad \text { for all } x \in \mathbf{R}^{d}, \quad \text { and }
$$




$$
m_{1}^{2} \stackrel{\text { def }}{=} \sup _{x \in \mathbf{R}^{d}} \sum_{j \in J}\left|\nabla \chi_{j}(x)\right|^{2}<+\infty, \quad m_{2}^{2} \stackrel{\text { def }}{=} \sup _{x \in \mathbf{R}^{d}} \sum_{j \in J}\left(\Delta \chi_{j}(x)\right)^{2}<+\infty
$$

Then the following estimates hold for any $u \in C_{0}^{\infty}\left(\mathbf{R}^{d}\right)$

$$
2\|Q u\|^{2}+3 m_{2}^{2}\|u\|^{2}+8 m_{1}^{2}\|\nabla u\|^{2} \geq \sum_{j \in J}\left\|Q \chi_{j} u\right\|^{2} .
$$

In particular, if $\Re V(x) \geq 0$,

$$
\begin{gathered}
2\|Q u\|^{2}+3 m_{2}^{2}\|u\|^{2}+8 m_{1}^{2} \Re\langle Q u, u\rangle \geq \sum_{j \in J}\left\|Q \chi_{j} u\right\|^{2}, \\
\langle Q u, u\rangle_{L^{2}}+m_{1}^{2}\|u\|^{2} \geq \sum_{j \in J}\left\langle Q \chi_{j} u, \chi_{j} u\right\rangle_{L^{2}} .
\end{gathered}
$$

Using a dyadic partition of unity, we apply Lemma 1 to the one-dimensional operator $Q=H_{\varepsilon}-i \lambda$.

LEMMA 2. For $j \in \mathbf{N}, \varepsilon>0$, and $\lambda \in \mathbf{R}$, we define unitary operators $U_{j}, j \in \mathbf{N}$ by $\left(U_{j} u\right)(x)=2^{j / 2} u\left(2^{j} x\right)$ and transform $Q$ by $U_{j}$

$$
P_{j, \varepsilon, \lambda}=U_{j} Q U_{j}^{*}=-2^{-2 j} \partial_{x}^{2}+2^{2 m j} x^{2 m}+\frac{i}{\varepsilon} f\left(2^{j} x\right)-i \lambda,
$$

and let

$$
C_{j}(\varepsilon, \lambda)=\inf \left\{\left\|P_{j, \varepsilon, \lambda} u\right\|: u \in C_{0}^{\infty}(\mathbf{R}), \operatorname{supp} u \subset K_{j},\|u\|=1\right\},
$$

where $K_{0}=[-1,1]$ and $K_{j}=[-1,-3 / 8] \cup[1,3 / 8]$ for any $j>0$. Then $\kappa(\varepsilon, \lambda)=$ $\left\|\left(H_{\varepsilon}-i \lambda\right)^{-1}\right\|$ satisfies

$$
\left(\inf _{j \in \mathbf{N}} C_{j}(\varepsilon, \lambda)\right)^{-1} \leq \kappa(\varepsilon, \lambda) \leq C\left(\inf _{j \in \mathbf{N}} C_{j}(\varepsilon, \lambda)\right)^{-1}
$$

for some constant $C \geq 1$ independent of $\varepsilon, \lambda$.

It is clear that $C_{j}(\varepsilon, \lambda) \geq a_{0}$ for all $j \in \mathbf{N}, \varepsilon>0, \lambda \in \mathbf{R}$, because

$$
a_{0}\|u\|^{2} \leq \Re\left\langle P_{j, \varepsilon, \lambda} u, u\right\rangle \leq\left\|P_{j, \varepsilon, \lambda} u\right\|\|u\|, \text { for all } u \in C_{0}^{\infty}(\mathbf{R}) .
$$

We now begin the proof of Proposition 1.

2.1. Proof of Proposition 1. (i) If $\operatorname{dist}(\varepsilon \lambda, f(\mathbf{R})) \geq \delta$, then

$$
\left|\Im\left\langle\left(H_{\varepsilon}-i \lambda\right) u, u\right\rangle\right|=\left|\left\langle\left(\frac{f}{\varepsilon}-\lambda\right) u, u\right\rangle\right| \geq(\delta / \varepsilon)\|u\|^{2} \quad \text { for all } u \in \mathcal{D},
$$


and we get $\kappa(\varepsilon, \lambda) \leq \varepsilon / \delta$. Before we prove (ii), for $f$ satisfying Hypothesis 1 , set that

$$
C_{f} \stackrel{\text { def }}{=} \sup _{j \in \mathbf{N}} \sup _{x \in K_{j}} 2^{k j}\left|f\left(2^{j} x\right)\right|<+\infty
$$

where $k>0$ is the parameter that governs the asymptotic behavior of $f(x)$ as $|x| \rightarrow \infty$.

(ii) Suppose that $\operatorname{dist}(\varepsilon \lambda, \operatorname{cv}(f) \cup\{0\}) \geq \delta$. We also assume that $\varepsilon|\lambda| \leq\|f\|_{L^{\infty}}+\delta$, because otherwise we can use the estimate (i). For any $u \in C_{0}^{\infty}(\mathbf{R})$ with supp $u \subset K_{j}$ and $u \not \equiv 0$, we have the lower bound

$$
\frac{\left\|P_{j, \varepsilon, \lambda}\right\|}{\|u\|} \geq \frac{\left|\Im\left\langle P_{j, \varepsilon, \lambda} u, u\right\rangle\right|}{\|u\|^{2}}=\frac{\left|\left\langle\left[2^{k j} f\left(2^{j} \cdot\right)-2^{k j} \varepsilon \lambda\right] u, u\right\rangle\right|}{\varepsilon 2^{k j}\|u\|^{2}} \geq \frac{1}{\varepsilon}\left(\varepsilon|\lambda|-\frac{C_{f}}{2^{k j}}\right) .
$$

Since $\varepsilon|\lambda| \geq \delta$, taking large enough $J \in \mathbf{N}$ such that $2^{k J} \geq 2 C_{f} / \delta$, we find that $C_{j}(\varepsilon, \lambda) \geq$ $\delta /(2 \varepsilon)$ for all $j \geq J$.

Thus, we only consider $0 \leq j \leq J$ and the problem is reduced to finding a lower bound on $\left\|\left(H_{\varepsilon}-i \lambda\right) u\right\|$ when $u \in C_{0}^{\infty}\left(\left\{x \in \mathbf{R} ;|x|<R_{\delta}\right\}\right)$, for some $R_{\delta}>0$. On a bounded domain, we can neglect the bounded term $x^{2 m}$ in $H_{\varepsilon}$ and only consider the operator $\tilde{Q}=-\partial_{x}^{2}+\frac{i}{\varepsilon}(f(x)-\varepsilon \lambda)$. Thus the method of [3] for the case $m=1$ applies here to obtain $\kappa(\varepsilon, \lambda) \leq C \varepsilon^{2 / 3}$.

(iii) The assumption $\lim _{\varepsilon \rightarrow 0} \varepsilon \lambda(\varepsilon)=\alpha \in \operatorname{cv}(f) \backslash\{0\}$ implies that $\varepsilon|\lambda| \geq \delta$ for some fixed $\delta>0$ if $\varepsilon>0$ is small enough. Thus, we can reduce the analysis to a bounded domain as in (ii) and again the analysis of [3] for the case $m=1$ yields the statement (iii).

(iv) For any $j \geq 1$ and $u \in C_{0}^{\infty}(\mathbf{R})$ with supp $u \subset K_{j}=\left\{\frac{3}{8} \leq|x| \leq 1\right\}$, we have

$$
\begin{gathered}
\|u\|\left\|P_{j, \varepsilon, 0} u\right\| \geq\left|\Re\left\langle P_{j, \varepsilon, 0} u, u\right\rangle\right| \geq 2^{2 m j} \int_{K_{j}}|x|^{2 m}|u(x)|^{2} d x \geq 3^{2 m} 2^{2(j-3) m}\|u\|^{2}, \\
\|u\|\left\|P_{j, \varepsilon, 0} u\right\| \geq\left|\Im\left\langle P_{j, \varepsilon, 0} u, u\right\rangle\right| \geq \frac{1}{\varepsilon 2^{k j}} \int_{K_{j}} 2^{k j}\left|f\left(2^{j} x\right)\left\|\left.u(x)\right|^{2} d x \geq \frac{m_{j}}{\varepsilon 2^{k j}}\right\| u \|^{2},\right.
\end{gathered}
$$

where $m_{j}(x)=\inf \left\{2^{k j}\left|f\left(2^{j} x\right)\right| ; \frac{3}{8} \leq|x| \leq 1\right\}$. From Hypothesis 1, we find that $\lim _{j \rightarrow \infty} m_{j}=1$, so taking large enough $J \in \mathbf{N}$, we find that

$$
C_{j}(\varepsilon, 0) \geq C\left(2^{m j}+\frac{1}{\varepsilon 2^{k j}}\right) \geq C \varepsilon^{-\frac{2 m}{k+2 m}}, \text { for all } j \geq J .
$$

Since $0 \leq j \leq J$ corresponds to a bounded spatial domain, we can treated as in (ii) and (iii). Hence, we find that

$$
\left\|H_{\varepsilon} u\right\| \geq C \varepsilon^{-\sigma}\|u\|, \quad \text { where } \sigma= \begin{cases}1, & \text { if } 0 \notin f(\mathbf{R}), \\ \frac{2}{3}, & \text { if } 0 \in f(\mathbf{R}) \backslash \operatorname{cv}(f), \\ \frac{1}{2}, & \text { if } 0 \in \operatorname{cv}(f) .\end{cases}
$$


Consequently, we get $\kappa(\varepsilon, 0) \leq C \varepsilon^{\min \left\{\frac{2 m}{k+2 m}, \sigma\right\}}$.

(v) By Lemma 2, we need only prove that

$$
C_{j}(\varepsilon, \lambda) \geq C \varepsilon^{-\min \left\{\frac{2 m}{k+3 m+1}, \frac{1}{2}\right\}}, \quad \text { for all } j \in \mathbf{N}, \quad 0<\varepsilon \ll 1 \quad \text { and } \quad \lambda \in \mathbf{R} .
$$

As in (ii), (iii), we have $C_{j}(\varepsilon, \lambda) \geq C_{J} \varepsilon^{-1 / 2}$ for $0 \leq j \leq J$. Hence, we consider the case $j>J$. We take $\tilde{u} \in C_{0}^{\infty}(\mathbf{R})$ such that $\operatorname{supp} \tilde{u} \subset K_{j},\|\tilde{u}\|=1$ and $\left\|P_{j, \varepsilon, \lambda} \tilde{u}\right\| \leq 2 C_{j}(\varepsilon, \lambda)$. As in (iv), we easily find that

$$
\left\|P_{j, \varepsilon, \lambda} \widetilde{u}\right\| \geq C 2^{2 m j}, \quad \text { and } \quad\left\|P_{j, \varepsilon, \lambda} \widetilde{u}\right\| \geq \frac{\inf _{x \in K_{j}}\left|g_{j}(x)\right|}{\varepsilon 2^{k j}},
$$

where

$$
g_{j}(x)=2^{k j} f\left(2^{j} x\right)-2^{k j} \varepsilon \lambda
$$

If $2^{j} \geq \varepsilon^{-\frac{1}{k+3 m+1}}$, the first inequality of (4) implies (3). If $2^{j}<\varepsilon^{-\frac{1}{k+3 m+1}}$, we integrate by parts and obtain the following relation:

$\left\|P_{j, \varepsilon, \lambda} \widetilde{u}\right\|^{2}+C 2^{2(m-1) j}\left\|x^{m-1} \widetilde{u}\right\|^{2}=\left\|Q_{j, \varepsilon, \lambda} \widetilde{u}\right\|^{2}+2^{2(m-1) j+1}\left\|x^{m} \partial_{x} \widetilde{u}\right\|^{2}+2^{4 m j}\left\|x^{2 m} \widetilde{u}\right\|^{2}$,

where $Q_{j, \varepsilon, \lambda}=P_{j, \varepsilon, \lambda}-2^{2 m j} x^{2 m}$. Thus, we have $\left\|P_{j, \varepsilon, \lambda} \tilde{u}\right\| \geq\left\|Q_{j, \varepsilon, \lambda} \tilde{u}\right\|-C 2^{(m-1) j}$. Combining this estimate with (4), we obtain

$$
2 C_{j}(\varepsilon, \lambda) \geq\left\|P_{j, \varepsilon, \lambda} \widetilde{u}\right\| \geq \frac{C}{3}\left(2^{2 m j}+\frac{\inf _{x \in K_{j}}\left|g_{j}(x)\right|}{\varepsilon 2^{k j}}+\left\|Q_{j, \varepsilon, \lambda} \widetilde{u}\right\|-2^{(m-1) j}\right) .
$$

As is proved by [3], we have

$$
\left\|Q_{j, \varepsilon, \lambda} u\right\| \geq \frac{C h^{2 / 3}}{\varepsilon 2^{k j}}\|u\|, \quad \text { for all } u \in C_{0}^{\infty}(\mathbf{R}) \quad \text { with supp } u \subset K_{j} .
$$

Returning to (5), we find that

$$
C_{j}(\varepsilon, \lambda) \geq C\left(2^{2 m j}+\frac{h^{2 / 3}}{\varepsilon 2^{k j}}-2^{(m-1) j}\right) \geq C \varepsilon^{\frac{-2 m}{k+3 m+1}},
$$

which proves (3).

2.2. Proof of Theorem 1. According to (v) in Proposition 1, it is clear that $\Psi(\varepsilon)=$ $\left(\sup _{\lambda \in \mathbf{R}} \kappa(\varepsilon, \lambda)\right)^{-1} \geq C^{-1} \varepsilon^{-v(m)}$. Since $\Sigma(\varepsilon) \geq \Psi(\varepsilon)$, we find that $\Sigma(\varepsilon) \geq C^{-1} \varepsilon^{-v(m)}$. Hence, we need only prove $\Psi(\varepsilon) \leq C \varepsilon^{-v(m)}$. First, we consider the case $k>m-1$. Fix $0<\varepsilon \ll 1,3 / 8<x_{0}<1$. We define $j \in \mathbf{N}, \lambda \in \mathbf{R}$ and $h>0$ as follows:

$$
2^{j} \geq \varepsilon^{-\frac{1}{k+3 m+1}}>2^{j-1}, \quad h^{2}=\varepsilon 2^{(k-2) j}, \quad \varepsilon \lambda=f\left(2^{j} x_{0}\right) .
$$


Next, we take $v \in C_{0}^{\infty}(\mathbf{R})$ such that $\|v\|=1$ and supp $v \subset[-1,1]$. We define

$$
u_{h}(x)=\frac{1}{h^{1 / 3}} v\left(\frac{x-x_{0}}{h^{2 / 3}}\right), \quad x \in \mathbf{R} .
$$

It is clear that $u_{h} \in C_{0}^{\infty}(\mathbf{R}),\left\|u_{h}\right\|=1$ and supp $u_{h} \subset K_{j}$ for sufficiently small $h>0$. Recalling that

$$
P_{j, \varepsilon, \lambda}=\frac{1}{\varepsilon 2^{k j}}\left(-h^{2} \partial_{x}^{2}+h^{2 / 3} x^{2 m}+i g_{j}(x)\right), \quad \text { where } g_{j}(x)=2^{k j} f\left(2^{j} x\right)-2^{k j} \varepsilon \lambda,
$$

we find that there exists $C>0$ independent of $j, \varepsilon, \lambda$ such that

$$
\left\|P_{j, \varepsilon, \lambda} u_{h}\right\| \leq C \frac{h^{2 / 3}}{\varepsilon 2^{k j}}=C \varepsilon^{-\frac{2 m}{k+3 m+1}} .
$$

This implies that $C_{j}(\varepsilon, \lambda) \leq C \varepsilon^{-\frac{2 m}{k+3 m+1}}$, hence $\kappa(\varepsilon, \lambda) \geq C \varepsilon^{\frac{2 m}{k+3 m+1}}$ by $(2)$ and $\Psi(\varepsilon) \leq$ $C \varepsilon^{-\frac{2 m}{k+3 m+1}}$. It is straightforward to verify (7). First, using (6), we find $\left\|h^{2} \partial_{x}^{2} u_{h}\right\|=h^{2 / 3}\left\|v^{\prime \prime}\right\|$. Next, since $x^{2 m} \leq x_{0}^{2 m}+2 m\left|x-x_{0}\right|$ for all $x \in K_{j}$, we have $\left\|x^{2 m} u_{h}\right\| \leq C$. Finally, since $g_{j}\left(x_{0}\right)=0$ by our choice of $\lambda$, we have for all $x \in K_{j}$,

$$
\left|g_{j}(x)\right| \leq\left|x-x_{0}\right| \sup _{\frac{3}{8} \leq|x| \leq 1}\left|g_{j}^{\prime}(x)\right| \leq C\left|x-x_{0}\right|,
$$

where $C$ does not depend on $j$ by Hypothesis 1 . Therefore, $\left\|g_{j} u_{h}\right\| \leq C h^{2 / 3}$ and the proof of (7) is complete.

Secondary, we consider the case $k \leq m-1$. Let $x_{0}$ be a critical point of $f$. We assume without loss of generality that $x_{0}=0$. We set

$$
\lambda=\frac{f(0)}{\varepsilon}, \quad g(x)=f(x)-\varepsilon \lambda .
$$

Next, we take $v \in C_{0}^{\infty}(\mathbf{R})$ such that $\|v\|=1$ and $\operatorname{supp} v \subset[-1,1]$. We define

$$
u_{\varepsilon}(x)=\frac{1}{\varepsilon^{1 / 8}} v\left(\frac{x}{\varepsilon^{1 / 4}}\right) .
$$

Using Taylor's expansion of $g$ around $x_{0}=0$, we find that

$$
\begin{aligned}
\left\|\left(H_{\varepsilon}-i \lambda\right) u_{\varepsilon}\right\| & \leq\left\|u_{\varepsilon}{ }^{\prime \prime}\right\|+\left\|x^{2 m} u_{\varepsilon}\right\|+\varepsilon^{-1}\left\|g u_{\varepsilon}\right\| \\
& =C \varepsilon^{-1 / 2}+C+C\left\|x^{2} u_{\varepsilon}\right\|+\mathcal{O}\left(\int_{\operatorname{supp} u_{\varepsilon}} x^{6}\left|u_{\varepsilon}(x)\right|^{2} d x\right)^{1 / 2} \\
& \leq C \varepsilon^{-1 / 2}
\end{aligned}
$$

Hence, $C^{-1} \varepsilon^{1 / 2} \leq \sup _{\lambda \in \mathbf{R}}\left\|\left(H_{\varepsilon}-i \lambda\right)^{-1}\right\|$ and we obtain $\Psi(\varepsilon) \leq C \varepsilon^{-1 / 2}$. 


\section{Spectral Lower Bounds - Proof of Theorem 2}

I. Gallagher, T. Gallay and F. Nier [3] have proved Theorem 2 for the case $m=1$, by using a complex deformation method and the same localization techniques as in the proof of Proposition 1. They also use accurate numerical computations to show that the lower bound in Theorem 2 is optimal when $m=1$, in the sense that the exponent $v^{\prime}(m)$ cannot be improved. Our proof for the general case follows that of Theorem 1.9 of [3]. We only give an outline the proof of Theorem 2 .

To prove Theorem 2, we use a complex deformation method using the dilation group $\left(U_{\theta} \phi\right)(x)=e^{\theta / 2} \phi\left(e^{\theta} x\right)$, which are unitary operators when $\theta \in \mathbf{R}$. If $f$ is given by $f(x)=$ $\left(1+x^{2}\right)^{-k / 2}$, the multiplication operator $(i / \varepsilon) f(x)$ is a dilation analytic perturbation of $H_{\infty}=$ $-\partial_{x}^{2}+x^{2 m}$. According to the dilation analytic theory ([4]), when we define the operator $H_{\varepsilon}(\theta)$ by

$$
H_{\varepsilon}(\theta)=U_{\theta} H_{\varepsilon} U_{\theta}^{-1}=-e^{-2 \theta} \partial_{x}^{2}+e^{2 m \theta} x^{2 m}+\frac{i}{\varepsilon} \frac{1}{\left(1+e^{2 \theta} x^{2}\right)^{k / 2}},
$$

for $S=\{\theta \in \mathbf{C} ;|\Im(\theta)| \leq \pi / 4 m\}$, the spectrum of $H_{\varepsilon}(\theta)$ does not depend on $\theta \in S$. We choose $\theta=i t_{k}$ where $t_{k}=\frac{\pi}{4 m(k+2)}$. Applying localization formula (1) in Lemma 1 to the operator $H_{\varepsilon}\left(i t_{k}\right)$, we obtain that

$$
\sigma\left(H_{\varepsilon}\right) \cap\left\{z \in \mathbf{C} ; c_{1} \Re(z) \leq|\Im(z)| \leq \frac{c_{2}}{\varepsilon}\right\}=\emptyset, \quad \text { for some } c_{1}, c_{2}>0 .
$$

As is proved by [3], combining this relation with the resolvent estimate of Proposition 1, we deduce that there exists $C>0$ such that $H_{\varepsilon}$ has no spectrum in the region $\left\{\Re(z) \leq C \varepsilon^{-v^{\prime}(m)}\right\}$ for sufficiently small $\varepsilon$. Therefore, we find that $\Sigma(\varepsilon) \geq C \varepsilon^{-v^{\prime}(m)}$ and this concludes the proof of Theorem 2.

ACKNOWLEDGMent. I would like to express sincere thanks to Professor Kenji Yajima for his unceasing encouragement and valuable advice. I also would like to thank the referee for giving me valuable comments.

\section{References}

[ 1] C. Villani, Hypocoercivity, Memoirs of the AMS.

[ 2 ] C. Villani, Hypocoercive diffusion operators, International Congress of Mathematicians, vol. 3, European Mathematical Society, Zürich, (2006), 473-498.

[ 3 ] I. Gallagher, T. Gallay and F. Nier, Spectral Asymptotics for Large Skew-Symmetric Perturbations of the Harmonic Oscillator, International Mathematics Research Notices (2009), 2147-2199.

[ 4] J. Aguilar and J. M. Combes, A class of analytic perturbations for one-body Schrödinger Hamiltonians, Communications in Mathematical Physics 22 (1971), 269-279.

[ 5 ] J. H. SCHENKER, Estimating Complex Eigenvalues of Non-Self Adjoint Schrödinger Operators via Complex Dilations, Mathematical Research Letters 18 (2011), no 04, 755-765. 
[ 6] T. Kato, Perturbation Theory for Linear Operators, Grundlehren der mathematischen Wissenschaften 132, Berlin, Springer, 1966.

Present Address:

DEPARTMENT OF MATHEMATICS,

GAKUSHUIN UNIVERSITY,

MEJIRO, TOSHIMA-KU, TOKYO, 171-8588 JAPAN.

e-mail: aiba@math.gakushuin.ac.jp 